\title{
Tendencias divergentes en la mortalidad por cáncer de próstata en la Comunidad Autónoma de Andalucía (1975-2004)
}

\author{
Cayuela Domínguez A*, Rodríguez-Domínguez $\mathrm{S}^{* *}$, Vigil Martín E, Medina López $\mathrm{R}^{* * *}$, \\ Conde Sánchez M.*** \\ Servicio de Documentación Clínica. Hospitales Universitarios Virgen del Rocío. Sevilla. **Fundación Progreso y \\ Salud. Sevilla. ${ }^{* * *}$ Servicio de Urología. Hospitales Universitarios Virgen del Rocío. Sevilla \\ Actas Urol Esp. 2007;31(2):92-97
}

\section{RESUMEN}

\section{TENDENCIAS DIVERGENTES EN LA MORTALIDAD POR CÁNCER DE PRÓSTATA EN LA COMUNIDAD AUTÓNOMA DE ANDALUCÍA (1975-2004)}

Objetivo. Describir la evolución de la mortalidad por cáncer de próstata en Andalucía durante el periodo 1975-2004 a nivel provincial.

Sujetos y método. Los datos de mortalidad por cáncer de próstata y las poblaciones necesarias para el cálculo de los indicadores fueron facilitados por el Instituto de Estadísticas de Andalucía. Se calcularon las tasas especificas por grupos de edad y estandarizadas globales y truncadas (35-64 años) mediante el método directo (población estándar europea).

Resultados. En el año 2004 se produjeron en Andalucía 813 defunciones por cáncer de próstata, representando el 8\% de la mortalidad por tumores. El análisis "joinpoint” permite delimitar tres tendencias diferenciadas en los últimos años (descenso en Cádiz, Huelva y Málaga, incremento en Granada y estabilización en el resto de provincias) en las tasas ajustadas de mortalidad por cáncer de próstata.

Conclusión. En Granada, en contra de la tendencia observada en el resto de provincias de Andalucía, se está produciendo un incremento en la mortalidad por cáncer de próstata.

Palabras clave: Cáncer de próstata. Mortalidad. Tendencia. Epidemiología.

\section{ABSTRACT}

DIVERGING TRENDS IN PROSTATE CANCER MORTALITY IN THE AUTONOMOUS COMMUNITY OF ANDALUCIA (1975-2004)

Objective. To describe the evolution of prostate cancer mortality in Andalucia during the period 1975-2004 at the provincial level.

Subjects and method. Mortality data for prostate cancer and population denominator data was supplied by the Statistical Institute of Andalucia. Age group-specific and standardised overall and truncated (50-79 years old) rates were calculated by the direct method (European standard population).

Results. In 2004 there were 813 deaths in Andalucia from prostate cancer. Prostate cancer accounts for around $8 \%$ of male deaths from cancer. Joinpoint analysis shows three diverging trends (fall in Cadiz, Huelva and Malaga, rise in Granada and stabilization in the other provinces) in age-standardized mortality rates.

Conclusion. In Granada, an increment in mortality from prostate cancer is observed in opposition to the trend in the other provinces. 
Q e estima que uno de cada seis varones desaOrrollará cáncer de próstata a lo largo de su vida $^{1}$.

En España, la mortalidad por cáncer de próstata experimentó un leve pero mantenido aumento entre los años 1951 y 1970 (3,32\% anual); desde entonces hasta mediados de los ochenta la mortalidad se estabiliza ${ }^{2-4}$ y permanece estable hasta 1994 donde comienza a descender $(-1,8 \%$ anual) ${ }^{5}$.

En trabajos previos se ha analizado la tendencia de la mortalidad por cáncer de próstata en Andalucía de forma global en el periodo 1975$1992^{6}$ y 1975-20017. Ambos estudios obtienen un descenso anual de -0,2\%, basándose en cálculos que asumen que las tasas aumentan o disminuyen con el tiempo a una tasa constante, aunque la validez de dicha asunción no fue evaluada ${ }^{8}$.

El modelo de regresión "joinpoint”, recientemente desarrollado, es útil para identificar y describir la ocurrencia de cambios en distintos periodos de tiempo a lo largo de la tendencia de los datos ${ }^{9,10}$. Teniendo en cuenta esto, nos planteamos proporcionar información actualizada y analizar los cambios en la mortalidad por cáncer de próstata a nivel de la Comunidad Autónoma de Andalucía y a nivel provincial en el período 1975-2004, usando modelos de regresión "joinpoint".

\section{PACIENTES Y MÉTODOS}

La información sobre las defunciones ocurridas en cada provincia de Andalucía se ha obtenido a partir de la información contenida en los boletines estadísticos de defunción (BED) grabada por el Instituto de Estadísticas de Andalucía (IEA) y suministrada en soporte magnético el período 1975-2004.

En el IEA se procede a la selección y codificación de la causa básica de defunción y se realizan diversos controles de calidad sobre la codificación de los diagnósticos médicos de las defunciones: recuperación de información sanitaria, si el BED no llega correctamente cumplimentado; revisión de la codificación, controles de validación de la causa básica de defunción con las variables demográficas, asignación de un código de control de calidad a cada BED, etc.
Estas defunciones se clasifican atendiendo a la causa básica de defunción y siguiendo la lista abreviada de la $8^{\mathrm{a}}, 9^{\mathrm{a}}$ y $10^{\mathrm{a}}$ Clasificación Internacional de Enfermedades y Causas de Muerte (CIE) (código 185 de la $8^{\underline{a}}$ y $9^{a}$ revisión de la CIE y código C61 de la $10^{-}$revisión de la $\mathrm{CIE}$ ).

Las poblaciones necesarias para el cálculo de los indicadores fueron facilitados por el Instituto de Estadísticas de Andalucía. Se han calculado las tasas estandarizadas global y truncada (3564 años) por el método directo (usando como estándar la población europea) expresadas como tasas por 100.000 varones-año.

Para estimar los cambios en la tendencia observados a lo largo del tiempo se han utilizado modelos segmentados de regresión de Poisson, llamados también "joinpoint regression analy-

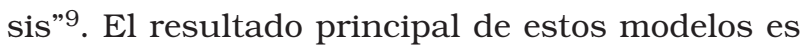
doble: identifican el momento en el que se producen cambios significativos en la tendencia y estiman la tendencia observada en cada intervalo correspondiente. En este tipo de análisis se eligen los puntos de mejor ajuste en los que la tasa cambia significativamente (aumenta o disminuye). El análisis empieza con el mínimo número de "joinpoints" y contrasta si uno o más de éstos son estadísticamente significativos y deben agregarse al modelo. En el modelo final, cada "joinpoint" indica un cambio estadísticamente significativo en la tendencia, y se calcula el porcentaje de cambio anual para cada una de esas tendencias por medio de modelos lineales generalizados asumiendo una distribución de Poisson. Los cambios significativos incluyen modificaciones en la dirección o en la tasa de aumento o disminución.

El cálculo de las tasas de mortalidad y sus errores estándar fue realizado en una hoja de cálculo (Microsoft $₫$ Excel). Los análisis “joinpoint” se realizaron mediante el software 'Joinpoint' del Surveillance Research Program of the US National Cancer Institute ${ }^{11}$.

\section{RESULTADOS}

El número de muertes por cáncer de próstata en Andalucía desde el año 1971 al 2004 aumentó de 407 a 813. En el año 2004, el cáncer de próstata representó el 8,0\% de la mortalidad por tumores en Andalucía. 
La Tabla 1 muestra los resultados del análisis de regresión “joinpoint”, es decir, los puntos en los que las tasas cambian significativamente y el porcentaje de cambio anual de cada tendencia según grupos de edad y áreas geográficas estudiadas.
Por grupos de edad, la regresión joinpoint realizada con modelo Poisson muestra una tendencia constante al descenso o estabilización en las tasas, por debajo de los 80 años. El grupo de 80 y más años de edad muestra dos periodos de incremento significativo (1975-1982 y 1989-1996

Tabla 1

Análisis joinpoint de las tasas de mortalidad por cáncer de próstata en Andalucía (1975-2004)

\begin{tabular}{|c|c|c|c|c|c|c|c|c|}
\hline \multirow[b]{2}{*}{$\begin{array}{l}\text { Tasas por } \\
\text { grupos de edad } \\
\text { (Andalucia) }\end{array}$} & \multicolumn{2}{|c|}{ Tendencia 1} & \multicolumn{2}{|c|}{ Tendencia 2} & \multicolumn{2}{|c|}{ Tendencia 3} & \multicolumn{2}{|c|}{ Tendencia 4} \\
\hline & Periodo & PCA $^{* *}$ & Periodo & PCA $^{* *}$ & Periodo & PCA** & Periodo & PCA** $^{* *}$ \\
\hline $50-59$ & $1975-2004$ & $-0,64$ & & & & & & \\
\hline $60-69$ & $1975-2004$ & $-0,56^{*}$ & & & & & & \\
\hline $70-79$ & $1975-2004$ & $-0,98^{*}$ & & & & & & \\
\hline $80+$ & 1975-1982 & $5,27^{*}$ & 1982-1989 & $-3,5^{*}$ & 1989-1996 & $3,6^{*}$ & 1996-2004 & $-0,8$ \\
\hline \multicolumn{9}{|l|}{ Tasas Brutas } \\
\hline Almería & $1975-2004$ & $1,95^{*}$ & & & & & & \\
\hline Cádiz & $1975-2004$ & $1,09 *$ & & & & & & \\
\hline Córdoba & 1975-2004 & $2,05^{*}$ & & & & & & \\
\hline Huelva & $1975-2004$ & $0,92^{*}$ & & & & & & \\
\hline Jaén & $1975-2004$ & $2,14^{*}$ & & & & & & \\
\hline Málaga & $1975-2004$ & $1,01^{*}$ & & & & & & \\
\hline Sevilla & $1975-2004$ & $1,90 *$ & & & & & & \\
\hline Andalucía & 1975-1983 & $2,6^{*}$ & 1983-1991 & 0,1 & 1991-1996 & $4,8^{*}$ & 1996-2004 & 0,4 \\
\hline \multicolumn{9}{|l|}{$\begin{array}{l}\text { Tasas Ajustadas } \\
\text { Global }\end{array}$} \\
\hline Almería & $1975-2004$ & 0,31 & & & & & & \\
\hline Cádiz & $1975-2004$ & $-0,99 *$ & & & & & & \\
\hline Córdoba & $1975-2004$ & $-0,02$ & & & & & & \\
\hline Granada & $1975-2004$ & $0,63^{*}$ & & & & & & \\
\hline Huelva & $1975-2004$ & $-0,65^{*}$ & & & & & & \\
\hline Málaga & $1975-2004$ & $-0,97^{*}$ & & & & & & \\
\hline Sevilla & $1975-2004$ & 0,02 & & & & & & \\
\hline Andalucía & 1975-1983 & 1,3 & 1983-1989 & $-2,8^{*}$ & 1989-1996 & 1,9 & 1996-2004 & $-1,1$ \\
\hline \multicolumn{9}{|l|}{ Truncada (35-64) } \\
\hline Almería & $1975-2004$ & 1,82 & & & & & & \\
\hline Cádiz & $1975-2004$ & $-1,46$ & & & & & & \\
\hline Córdoba & 1975-2004 & $-1,48$ & & & & & & \\
\hline Granada & $1975-2004$ & $2,29 *$ & & & & & & \\
\hline Huelva & $1975-2004$ & $-0,13$ & & & & & & \\
\hline Jaén & $1975-2004$ & 0,39 & & & & & & \\
\hline Málaga & $1975-2004$ & $-1,23$ & & & & & & \\
\hline Sevilla & 1975-2004 & $-0,78$ & & & & & & \\
\hline Andalucía & $1975-2004$ & $-0,6^{*}$ & & & & & & \\
\hline
\end{tabular}

**PCA: porcentaje de cambio anual estimado mediante análisis joinpoint *E1 PCA es significativamente distinto de $0(\mathrm{p}<0,05)$ 
incrementos del 5,3\% y del 3,6\% anual respectivamente), un periodo de descenso estadísticamente significativo (1982-1989 descenso del $3,5 \%)$ y un periodo final (1996-2004) donde las tasas se estabilizan (descenso del 0,8\% anual no significativo estadísticamente).

El análisis joinpoint nos permite diferenciar 2 periodos de incrementos significativos (19751983 y 1991-1996 con incrementos del 2,6\% y $4,8 \%$ anual respectivamente) y dos periodos de estabilización de las tasas (1983-1991 y 19962004 con incrementos inferiores al 0,5\% anual y no significativos estadísticamente) en las tasas brutas de mortalidad por cáncer de próstata en Andalucía. En todas las provincias se observa un incremento estadísticamente significativo en las tasas brutas que oscila entre el 0,92\% anual en Huelva y el 2,78\% anual en Granada.

Las tasas ajustadas (globales) por edad en Andalucía descienden a lo largo de todo el periodo de estudio $(-0,26 \%$ anual, $\mathrm{p}<0,05)$. Sin embargo el análisis joinpoint refleja cuatro periodos: 1975-1983 de incremento no significativo (1,3\% anual), 1983-1989 de descenso estadísticamente significativo (-2,8\% anual), 1989-1996 de incremento no significativo (1,9\% anual) y 1996-2004 de descenso no significativo (-1,1\% anual). A nivel de provincias se aprecian tres patrones: descenso estadísticamente significativo de las tasas (Cádiz, Málaga y Huelva), estabilización (incremento o decremento inferior al $0,5 \%$ anual) en Almería, Córdoba, Jaén y Sevilla e incremento estadísticamente significativo en Granada. El mayor descenso se produce en la provincia de Cádiz donde las tasas pasan de 27,2 por 100000 en el periodo $1975 / 77$ a 18,3 en el periodo 2000/04 (Tabla 2).

Las tasas ajustadas truncadas (35-64 años) muestran un paulatino descenso significativo del -0,6\% anual a nivel de Andalucía. Al analizar por provincias observamos que la única provincia que muestra una tendencia estadísticamente significativa es Granada (las según provincias tasas se incrementan un 2,3\% anual pasando de 3,2 por 100.000 en $1975 / 77$ a 5,3 en 2000/04) (Tablas 1 y 2) mientras que el resto de provincias muestran incrementos o decrementos no estadísticamente significativos.

\section{DISCUSIÓN}

El análisis de las tendencias de mortalidad tiene una larga y continua tradición en la investigación de resultados en salud y ha ayudado a comprender mejor los determinantes de la enfermedad $^{5}$. En nuestro medio, dada la escasa disponibilidad de datos sobre morbilidad, hemos tenido que usar los datos de mortalidad (fuente de datos que satisface los criterios de continuidad y globalidad $^{12}$ y la exactitud de los certificados de defunción con esta causa) ${ }^{13}$.

La certificación de la causa de muerte a edades avanzadas puede estar influenciada por las mejoras diagnósticas. A estas edades se encuentran focos neoplásicos en la próstata en una gran proporción de varones. Por ello, el análisis de las tendencias temporales debe basarse sobre todo en tasas a edades medias, aunque éstas representen una pequeña proporción de los casos.

En el año 2004, usando la base de datos de mortalidad de la OMS, Baade et $\mathrm{al}^{5}$ analizó la tendencia de las tasas ajustadas (grupo de 50-79 años de edad y población estándar europea) de mortalidad por cáncer de próstata en el periodo

Tasas de mortalidad por cáncer de próstata en Andalucía (1975/77 y 2000/04)

\begin{tabular}{lcccccc}
\hline & \multicolumn{2}{c}{ TB } & \multicolumn{2}{c}{ TE } & \multicolumn{2}{c}{ TT } \\
& $\mathbf{1 9 7 5 / 7 7}$ & $\mathbf{2 0 0 0 / 0 4}$ & $\mathbf{1 9 7 5 / 7 7}$ & $\mathbf{2 0 0 0 / 0 4}$ & $\mathbf{1 9 7 5 / 7 7}$ & $\mathbf{2 0 0 0 / 0 4}$ \\
\hline Almería & 12,6 & 19,5 & 17,7 & 17,9 & 5,3 & 3,7 \\
Cádiz & 13,5 & 16,6 & 27,2 & 18,3 & 7,4 & 4,2 \\
Córdoba & 11,8 & 21,7 & 15,5 & 15,8 & 3,7 & 2,2 \\
Granada & 13,0 & 23,5 & 17,7 & 18,3 & 3,2 & 5,3 \\
Huelva & 15,8 & 24,2 & 20,3 & 20,4 & 2,5 & 3,4 \\
Jaén & 14,3 & 24,7 & 17,1 & 17,1 & 3,0 & 3,6 \\
Málaga & 15,7 & 20,1 & 24,6 & 18,4 & 7,6 & 4,6 \\
Sevilla & 12,2 & 19,9 & 19,5 & 19,3 & 5,8 & 4,2 \\
& & & & & & \\
Andalucía & 13,5 & 20,9 & 21,0 & 20,0 & 5,3 & 4,3 \\
\hline
\end{tabular}

TB: Tasa bruta por 100.000 varones/año.

TE: Tasas ajustada por edad (todas las edades) por 100.000 varones/año.

TT: Tasa ajustada por edad (35-64 años) por 100.000 varones/año. 
1979-1999 y observo un descenso desde mediados de la década de los noventa en Inglaterra, Estados Unidos, Austria, Canadá, Italia, Francia, Alemania, Austria, Holanda, Irlanda y España. Diferentes razones se han sugerido para explicar esas tendencias incluyendo las mejoras en el diagnóstico y tratamiento del cáncer de próstata. Usando la misma metodología nosotros observamos para el mismo periodo (1979-1999) un descenso continuado de 0,7\% anual (estadísticamente significativo).

En Andalucía, sin embargo, dicho descenso se viene observando de forma continuada desde $1975^{6,7}$ (Tabla 1, Fig. 1). Esta tendencia descendente se observa en casi todos los grupos de edad a lo largo del periodo de estudio lo que sugiere un efecto periodo (descrito también en otras áreas geográficas) ${ }^{14-16}$ (Tabla 1, Fig. 2). Pese a ello, al analizar a nivel de provincias vemos que esto solo sucede en Cádiz, Málaga y Huelva y que en Granada las tasas ajustadas se han incrementado de forma significativa sobre todo en el grupo de 35-64 años (incremento del 2,3\% anual).

En Granada (la única provincia en la Comunidad Autónoma de Andalucía que cuenta con un registro de tumores de base poblacional) las tasas de incidencia de cáncer de próstata se han incrementado un $5,3 \%$ anual durante el periodo 1985-2002. Los varones más jóvenes (grupo de 45-64 años) mostraron el mayor incremento en las tasas de incidencia $(11,1 \%)$ mientras que en los mayores de 64 este incremento fue inferior $(7,6 \%$ en el grupo de 64-74 años y $1,1 \%$ en los mayores de 75 años) ${ }^{17}$. Este comportamiento en las tasas de incidencia se estaría viendo reflejado en nuestros resultados en cuanto a la mortalidad (Tabla 2) con incrementos del 0,6\% y 2,3\% anual en las tasas ajustadas (global y truncada respectivamente) a lo largo del periodo 1975-2004. Además, un reciente trabajo muestra que Granada presenta un mayor porcentaje de pacientes con enfermedad localmente avanzada y metastásica que el resto de provincias ${ }^{18}$.

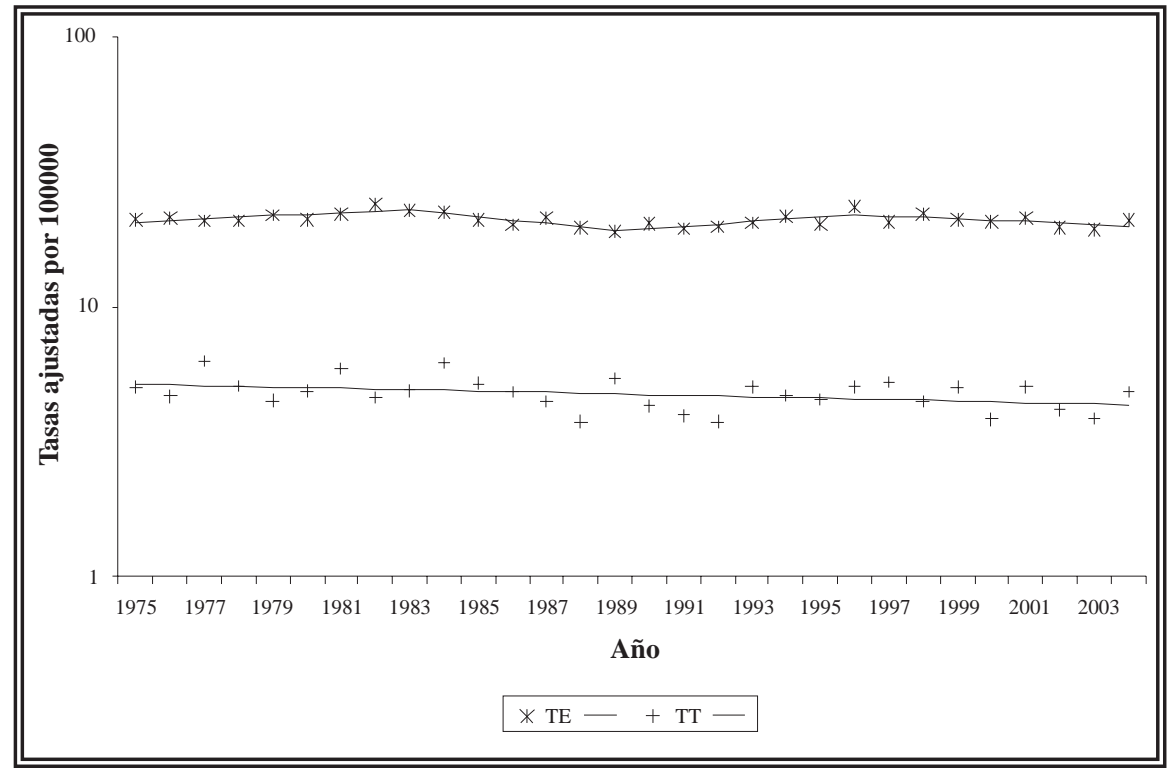

FIGURA 1. Tasas estandarizadas (por 100.000, población estándar Europea) de mortalidad por cáncer de próstata en Andalucía (1975-2004).

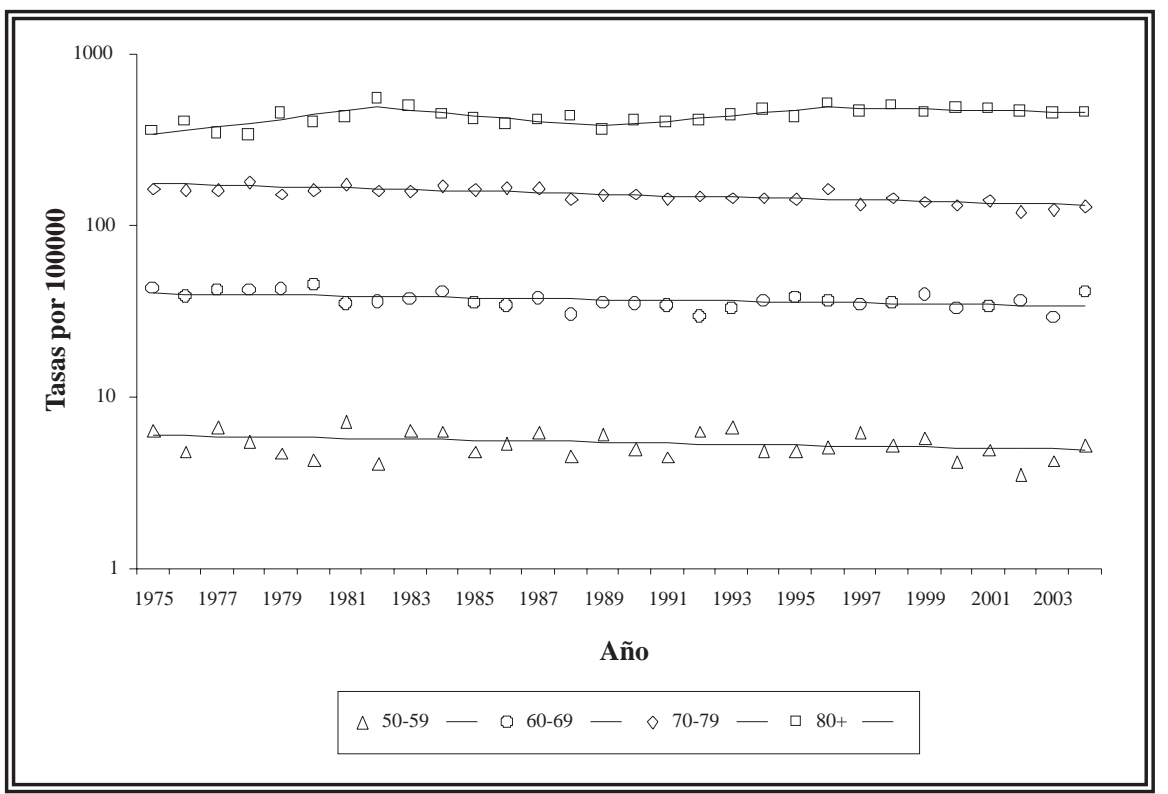

FIGURA 2. Mortalidad por cáncer de próstata en Andalucía (1975-2004). Tasas especificas por grupos de edad. 
En el año 2004 las tasas brutas de incidencia por cáncer de próstata en las provincias de la Comunidad Autónoma de Andalucía oscilaron entre 26,7 por 100.000 varones en Cádiz y 97,1 en Córdoba ${ }^{18}$. Desafortunadamente no tenemos información sobre la evolución de la incidencia de cáncer de próstata en el resto de provincias de Andalucía, aunque podemos pensar a partir de nuestros datos que en algunas de ellas (Cádiz, Huelva y Málaga) podría estar disminuyendo.

En conclusión podemos afirmar que el descenso en la mortalidad por cáncer de próstata se produce en Andalucía muchos años antes que en España y otros países de nuestro entorno. Pese a ello, el análisis "joinpoint" permite delimitar tres tendencias diferenciadas en los últimos años (descenso en Cádiz, Huelva y Málaga, incremento en Granada y estabilización en el resto de provincias andaluzas) en las tasas ajustadas de mortalidad por cáncer de próstata.

\section{REFERENCIAS}

1. Herranz F, Arias F, Arrizabalaga M, Calahorra FJ Carballido J, Diz R, et al. El cáncer de próstata en la Comunidad de Madrid en el año 2000. II- Presentación y diagnóstico. Actas Urol Esp. 2003;27(5):335-344.

2. Cayuela A, Lacalle JR, Perea E. Análisis de la mortalidad por cáncer de próstata. España 1951-1979. Oncología 1987;10(8):364-370.

3. Cayuela A, Lacalle JR, Gili M. Analysis of cohort mortality from prostatic cancer in Spain, 1951-1983. J Epidemiol Commun Health. 1989;43(3):249-252.

4. Bolumar F, Vioque J, Cayuela A. Changing mortality patterns for major cancers in Spain, 1951-1985. Int J Epidemiol. 1991;20(1):20-25.

5. Baade PD, Coory MD, Aitken JF. International trends in prostate-cancer mortality: the decrease is continuing and spreading. Cancer Causes and Control. 2004;15(3):237241 .
6. Cayuela A, Rodríguez S. Distribución de la mortalidad por cáncer de próstata. Andalucía 1975-1992. Rev Esp Geriatría y Gerontología 1996;31(4):217-225.

7. Ruiz-Ramos M, Escolar Pujolar A. La mortalidad por cáncer de próstata en andalucía: aportaciones al cribado poblacional. Actas Urol Esp. 2005;29(1):41-46.

8. Esteve J, Benhamou E, Raymond L. Statistical methods in cancer research. Volume IV. Descriptive Epidemiology. IARC Sci Publ. 1994;(128):301-302.

9. Kim H-J, Fay MP, Feuer EJ, Midthune DN. Permutation tests for joinpoint regression with applications to cancer rates. Stat Med. 2000;19(3):335-351.

10. Cayuela A, Rodriguez-Dominguez S, Lopez-Campos JL, Otero Candelera R, Rodriguez Matutes C. Joinpoint regression analysis of lung cancer mortality, Andalucia 19752000. Ann Oncol. 2004;15(5):793-796.

11. www-dccps.ims.nci.nih.gov/SRAB último acceso 10/08/ 2006.

12. Glasser JH. The quality and utility of death certificate data. Am J Public Health. 1981;71(3):231-233.

13. Martínez C, Sánchez MJ, Rodríguez M, Alamitos FJ, Medina MJ. Exactitud del diagnóstico de cáncer en los certificados de defunción de la provincia de Granada. Revista de Oncología. 2000;2(5):245-252.

14. Oliver SE, Gunnell D, Donovan JL. Comparison of trends in prostate-cancer mortality in England and Wales and the USA. Lancet 2000;355(9217):1788-1789.

15. Oliver S, May M, Gunnell D. International trends in prostate-cancer mortality in the "PSA ERA". Int $J$ Cancer. 2001;92(6):893-898.

16. Potosky AL, Feuer EJ, Levin DL. Impact of screening on incidence and mortality of prostate cancer in the United States. Epidemiol Rev. 2001;23(1):181-186.

17. Martínez C, Sánchez MJ, Rodríguez M, Chang YL. Incidente of prostate cancer in Granada 1998-2002 and time trends during the period 1985-2002. Available at: http://www.grellnet.org/2006/diapos/o29\%20maria\%20j $\% 20$ sanchez.pdf

18. Morales A. El cáncer de próstata en Andalucía año 2004. Asociación Andaluza de Urología. 2006.

Correspondencia autor: Dr. Aurelio Cayuela. Servicio de Documentación Clínica. Hospitales Universitarios Virgen del Rocío. Avda. de Manuel Siurot s/n. 41013-Sevilla (Spain). Tel.: +34955013299.

E-mail autor: aurelio.cayuela.sspa@juntadeandalucia.es Información artículo: Original - Revisión

Trabajo recibido: octubre 2006

Trabajo aceptado: noviembre 2006 